NBER WORKING PAPER SERIES

\title{
THE MAGNITUDE AND NATURE OF RISK SELECTION IN EMPLOYER-SPONSORED HEALTH PLANS
}

\author{
Sean Nicholson \\ M. Kate Bundorf \\ Rebecca M. Stein \\ Daniel Polsky \\ Working Paper 9937 \\ http://www.nber.org/papers/w9937 \\ NATIONAL BUREAU OF ECONOMIC RESEARCH \\ 1050 Massachusetts Avenue \\ Cambridge, MA 02138 \\ August 2003
}

We would like to thank the Robert Wood Johnson Foundation for supporting this research project. Helpful comments have been provided by Roger Feldman, Jeannette Rogowski, and participants of the Eighth Northeast Regional Health Economics Research Symposium, the International Health Economics meetings, and a conference at the University of California, Irvine: Competition and Consumer Choice in Health Insurance: An International Perspective. The views expressed herein are those of the authors and not necessarily those of the National Bureau of Economic Research.

(C)2003 by Sean Nicholson, M. Kate Bundorf, Rebecca M. Stein, and Daniel Polsky. All rights reserved. Short sections of text, not to exceed two paragraphs, may be quoted without explicit permission provided that full credit, including (C) notice, is given to the source. 
The Magnitude and Nature of Risk Selection in Employer-Sponsored Health Plans

Sean Nicholson, M. Kate Bundorf, Rebecca M. Stein, and Daniel Polsky

NBER Working Paper No. 9937

August 2003

JEL No. I11

\begin{abstract}
Most existing studies of risk selection in the employer-sponsored health insurance market are case studies of a single employer or of an employer coalition in a single market. We examine risk selection in the employer-sponsored market by applying a "switcher" methodology to a national, panel data set of enrollees in employer-sponsored health plans. We find that people who switched from a non-HMO to an HMO plan used 11 percent fewer medical services in the period prior to switching than people who remained in the nonHMO plan, and that this relatively low use persists once they enroll in an HMO. Furthermore, people who switch from an HMO to a non-HMO plan used 18 percent more medical services in the period prior to switching than those who remained in an HMO plan. HMOs would most likely continue to experience favorable risk selection if employers adjusted health plan payments based on enrollees' gender and age because the selection appears to occur based on enrollee characteristics that are difficult to observe, such as preferences for medical care and health status.
\end{abstract}

Sean Nicholson

The Wharton School

3641 Locust Walk

Philadelphia, PA 19104

and NBER

nicholss@wharton.upenn.edu
M. Kate Bundorf

Health Research and Policy

Stanford University

HRP T152

Stanford, CA 94305-5405

and NBER

bundorf@standford.edu

\section{Rebecca M. Stein}

Economics Department

University of Pennsylvania

3718 Locust Walk

Philadelphia, PA 19604

rstein2@ssc.upenn.edu
Daniel Polsky

General Internal Medicine

University of Pennsylvania

Blockley Hall, Room 1212

Philadelphia, PA 19104-6021

polsky@mail.med.upenn.edu 


\section{Introduction}

Managed care health plans currently cover about 90 percent of the people who receive employersponsored health insurance. Health insurance companies often have incentives to design their plans to attract low-risk enrollees and repel high-risk enrollees. It has been argued that a health insurance market providing both managed care and non-managed care plans may be inefficient because managed care plans may be more likely to implement such strategies, resulting in too few people in non-managed care plans (Cutler and Zeckhauser, 1998). In fact, a number of studies have shown that Medicare HMOs attract a disproportionate share of the healthy elderly population (Eggers, 1980; Eggers and Prihoda, 1982; Brown et al., 1993; Cox and Hogan, 1997; Call et al., 1999). Unlike the Medicare risk selection studies that are national in scope, most studies of risk selection in the employer-sponsored health insurance market examine single employers or employer coalitions, and the available evidence is mixed. Cutler and Zeckhauser (1998) found substantial favorable risk selection among state government employees in Massachusetts for the HMO plan relative to the fee-for-service plan. Altman, Cutler, and Zeckhauser (2003), examining the same data set, find that almost half of the expenditure difference between indemnity and HMO plans for eight medical conditions is due to a lower incidence rate among HMO patients (favorable risk selection), with the remaining difference due to lower provider reimbursement. In contrast, the RAND Health Insurance Experiment did not find that a Seattle HMO experienced a favorable selection of patients (Manning et al., 1987), and Polsky and Nicholson (2002) conclude that HMOs in 60 metropolitan areas were not experiencing favorable selection with respect to non-HMOs in 1996 and 1997.

Eighty percent of the non-elderly who had health insurance in 1999 received it from their employer or from a family member's employer. ${ }^{1}$ Risk selection can occur because only one percent of U.S. employers adjust the premium payment to a health plan based on an employee's risk, or expected

\footnotetext{
${ }^{1}$ Current Population Survey, 1999.
} 
medical expenditures (Kennan et al., 2001). ${ }^{2}$ As a result, most health insurance companies receive the same revenue for enrolling a young healthy worker as an old sick worker, although the expected medical costs of the two workers could differ substantially. If health insurers design their plans to attract the relatively profitable, low-risk enrollees, an inefficient allocation of individuals to plans may result. A plan that attracts low-risk enrollees can charge a lower premium than a plan that is experiencing adverse selection. As a result, some people who would enroll in a relatively expensive plan (usually a non-HMO) if premiums were risk-adjusted to reflect their unique expected costs, will enroll instead in a less expensive plan (usually an HMO) once the premium difference becomes sufficiently large (Cutler and Zeckhauser, 1998).

Even though few firms formally risk adjust premium payments to health plans, risk selection may still be limited. An estimated 20 percent of the employer-sponsored market is enrolled in self-insured plans where health insurance companies usually receive a fixed payment per employee to administer the plans and do not bear the financial risk of uncertain medical expenditures (McDonnell and Fronstin, 1999; InsterStudy, 2000). Furthermore, about 50 percent of the people insured in the employer-sponsored market were offered a plan or a choice of plans from a single insurance carrier (Keenan et al., 2001). In these two situations health insurers have no incentive to design plans to attract low-risk employees. Some firms may also be implementing implicit (to the analyst) risk adjustment by setting the employer's contribution for plans experiencing adverse selection higher than the employer's contribution for plans experiencing favorable risk selection. Such behavior would encourage all employees to enroll in the plan experiencing adverse selection.

With the exception of Polsky and Nicholson (2002), all of the studies cited above that examine the non-elderly, employer-sponsored market are case studies of a single employer or employer coalition in a single market. Since many employers have instituted measures to mitigate incentives for health plans to target low-risk enrollees, the results from the case studies mentioned above may not be representative

2 Medicare, on the other hand, does adjust payments to HMOs based on an enrollee's age, gender, county of residence, Medicaid eligibility, and whether or not they are institutionalized in a nursing home. 
of national conditions. In this paper we examine whether HMO plans experience favorable risk selection in the employer-sponsored market by applying a "switcher" methodology to the Community Tracking Study Household Survey (CTSHS), a recently released national, panel data set of enrollees in employersponsored health plans. The switcher methodology compares medical expenditures in the initial year of people who chose to switch from a non-HMO (a fee-for-service indemnity plan or a preferred provider organization) to an HMO plan versus the expenditures in the initial year of the people who remained in the non-HMO plan both years (and likewise for people who initially were enrolled in an HMO). In addition, we further decompose risk selection into a component based on enrollee characteristics that health plans and employers clearly observe (e.g., gender and age) and a component based on enrollee characteristics that are difficult to observe (e.g., preferences for medical care or health status). This decomposition will help determine whether risk adjusting peoples' premiums based on observed characteristics is likely to minimize or eliminate selection.

We find that HMOs are experiencing favorable risk selection and that this risk selection would probably persist even if employers adjusted premiums based on an enrollee's gender and age. People who switched from a non-HMO to an HMO plan used 11 percent fewer medical services in the period prior to switching than people who remained in non-HMO plans, and this relatively low use persists once they enroll in an HMO, relative to those already enrolled in an HMO. We also find that people who switched from an HMO to a non-HMO plan used 18 percent more medical services in the period prior to switching than people who remained in the HMO plan. This relatively high use rate does persist once they enroll in the non-HMO, but the differential is not statistically significant. Ten percent of HMO enrollees switched to a non-HMO between the first and second round of the CTSHS survey, and their departure is predicted to reduce an HMO's aggregate medical expenditures by 2.0 percent per year. Over a number of years, risk selection of this magnitude could allow HMOs to charge lower premiums than non-HMOs and attract enrollees who would prefer to be in non-HMOs if premiums could be perfectly risk adjusted. Favorable risk selection by HMOs would most likely persist even if employers adjusted 
health plan payments based on enrollees' gender and age, because the selection appears to occur based on enrollee characteristics that are difficult to observe, such as preferences for medical care and health status.

\section{Empirical Methodology}

We define risk selection as the difference in medical resource use between HMO enrollees and non-HMO enrollees due to differences in enrollees' characteristics, and therefore their demand for medical services, but not due to differences in the design and management of the two plan types. If we compare resource use for people who have chosen to enroll in an HMO to people who have chosen to enroll in a non-HMO plan, we cannot determine how much of the difference is due to enrollee characteristics and how much is due to differences in how the plans are designed and managed. We examine people for two years and compare first period medical resource use between people who were initially enrolled in a non-HMO and switch to an HMO in the second period to those who remain in a non-HMO plan, and likewise for people initially enrolled in an HMO. By comparing resource use when the two sets of individuals were enrolled in the same type of plan, expenditure differences can be attributed to differences in demand for medical services due to differences in health and preferences for medical care, rather than differences in cost sharing or the extent of the plans' utilization management efforts. Since we examine risk selection only for people who switch from a non-HMO to an HMO or from an $\mathrm{HMO}$ to a non-HMO, we estimate a marginal risk selection measure that is relevant for the enrollees switching plan types, rather than an average risk selection measure that would be relevant for all enrollees.

We derive separate estimates of risk selection for those who are initially enrolled in an HMO $(\mathrm{H})$ plan and for those who are initially enrolled in a non-HMO plan $(\mathrm{NH})$. We aggregate medical resource use into a single expenditure measure using unit prices for each type of service (e.g., price per hospital day, price per physician visit). Consider a sample of people who are observed in two time periods and were offered a choice of a non-HMO and an HMO plan by their employer in the second time period. We 
categorize people into four mutually exclusive groups according to the plan in which they were enrolled in the first and second periods: people who were enrolled in a non-HMO plan in both periods are coded as NH_NH, people who were enrolled in an HMO plan in both periods as $\mathrm{H} \_\mathrm{H}$, people who switched from a non-HMO to an HMO plan as NH_H, and people who switched from an HMO plan to a non-HMO plan as H_NH.

Using ordinary least squares, we regress medical expenditures for person $\mathrm{i}$ in the first period, measured in dollars $\left(\mathrm{Y}_{\mathrm{i} 1}\right)$, on the four indicator variables that characterize the type of plan in the first and second periods, and individual and firm characteristics $\left(\mathbf{X}_{\mathrm{i}}\right)$ that could potentially be observed by an employer and used to risk adjust premiums:

$$
\mathrm{Y}_{\mathrm{i} 1}=\beta_{1} \mathrm{NH}_{-} \mathrm{NH}_{\mathrm{i}}+\beta_{2} \mathrm{NH}_{-} \mathrm{H}_{\mathrm{i}}+\beta_{3} \mathrm{H}_{-} \mathrm{H}_{\mathrm{i}}+\beta_{4} \mathrm{H}_{-} \mathrm{NH}_{\mathrm{i}}+\beta_{5} \mathbf{X}_{\mathrm{i}}+\varepsilon_{\mathrm{i}}
$$

The difference in first period medical expenditures between those who switch to an HMO in the second period $\left(\mathrm{NH} \_\mathrm{H}=1\right)$ and those who remain enrolled in a non-HMO plan $\left(\mathrm{NH}_{-} \mathrm{NH}=1\right)$ is $\left(\beta_{2}-\beta_{1}\right)$. If this difference is negative and significantly different from zero, then HMO plans experience favorable risk selection relative to the non-HMO plans. Among the population who are not already enrolled in an HMO, HMOs attract people with relatively low medical expenditures. The difference in first period medical expenditures between those who switch to a non-HMO in the second period $\left(\mathrm{H} \_\mathrm{NH}=1\right)$ and those who remain enrolled in an HMO plan $\left(\mathrm{H}_{-} \mathrm{H}=1\right)$ is $\left(\beta_{4}-\beta_{3}\right)$. If this difference is positive and significantly different from zero, then non-HMO plans experience adverse risk selection relative to the HMO plans. Among the population who are not already enrolled in a non-HMO, non-HMOs attract people with relatively high medical expenditures.

If an employer uses observed characteristics (e.g., age, gender, and possibly health measures) to estimate an employee's expected medical expenditures and adjust the premium payments that a health plan receives, all employees will be equally profitable from a health insurer's perspective, ex ante. To explore this, we first estimate equation (1) with controls for the geographic site (usually a metropolitan area) but without any enrollee characteristics $\mathbf{X}$. We then estimate equation (1) with an indicator variable 
for an employee's gender and a set of indicators for their age. If $\left(\beta_{2}-\beta_{1}\right)$ and $\left(\beta_{4}-\beta_{3}\right)$ are significant without controlling for age and gender but insignificant once these characteristics are included in the regression, this implies that employers can mitigate risk selection by simply adjusting health plan payments for age and gender. That is, health plans would not have incentives to design their plans to attract people who are expected to be relatively low users of medical services, because the premiums would be adjusted such that the expected profit would not vary across employee types. We also estimate equation (1) with a more comprehensive set of possible risk adjusters such as self-reported health, income level, size of firm, marital status, and the presence of children to see if risk selection would persist with a more sophisticated risk adjustment system. This specification probably includes more information than an employer would have for purposes of adjusting premiums, but we present it as an extreme case. If risk selection is based on observable enrollee characteristics only, then health insurance premiums could be risk adjusted to prevent plans from designing their plans to attract the most profitable employees. If risk selection is based on unobserved characteristics, on the other hand, then other policies such as a patient bill of rights law that restrict plan design may be useful.

We restrict the samples in the above two regressions to people who could choose either an HMO or a non-HMO in the second period because we want to focus on situations where risk selection is most likely to occur. If a firm offers one type of plan to its workers from one health insurer, then it is relatively easy for the insurer to predict aggregate medical costs since it will enroll all employees. However, some people in our sample had a constrained choice of health plan types in the initial period -- they were either offered an HMO plan only or a non-HMO plan only -- and then had a choice of plan types in the second period. Among those unconstrained in their ability to choose their type of health plan in the initial observation period, much of the sorting of risk between managed care and non-managed care may have already taken place in previous years. To examine this, we create eight rather than four indicator variables in equation (1) - four variables for people without a choice of plan types in the first period (e.g., $\mathrm{H}_{-} \mathrm{H}^{\mathrm{NC}}$ for people who were only offered an HMO in the first period, and chose an HMO in the second period when they had a choice of plan types), and four variables for people who were offered both an 
HMO and a non-HMO by their employer in the first period (e.g., $\mathrm{H}_{-} \mathrm{NH}^{\mathrm{C}}$ for people who had a choice of plans in the first period and chose an HMO, and chose a non-HMO in the second period when they had a choice of plan types). The "NC" superscript refers to no choice and the "C" refers to a choice of plan types. We would expect more pronounced risk selection among those who move from a constrained environment ( $\mathrm{NC}$ in the first period) to an unconstrained environment relative to people who had a choice of plan types in both periods.

We also examine medical expenditures in the second period to see if people who switch plan types have higher or lower expenditures relative to the group of enrollees they join. If, for example, HMOs attract relatively low users of medical care among the people initially enrolled in non-HMO plans but these enrollees have second period-expenditures that are similar to those initially enrolled in an HMO, then risk selection may lower the profitability of non-HMOs but it will have little effect on the profitability of HMOs. This would be the case, for example, if the first period expenditure difference is due to a negative or positive health shock that is transitory, and does not persist in the second period. We run regressions similar to equation (1), where the dependent variable is an enrollee's medical expenditures in the second period, and the key variables are indicators for people who switched plan types between the first and second time periods.

\section{Data}

We use data from the first (1996-1997) and second (1998-1999) rounds of the Community Tracking Study Household Survey (CTSHS). Each survey was administered to more than 60,000 people and was designed to be representative of the civilian noninstitutionalized population in 60 U.S. communities and the country as a whole (Kemper et al., 1996). We focus our analysis on the 11,672 nonelderly persons who were surveyed in both rounds and received health insurance from their employer or the employer of a household member in both years of the survey. The regressions further restrict the sample to the 6,235 people who were offered both an HMO and a non-HMO plan by their employer in the second time period. The CTSHS attempted to re-survey a random subset of the round-1 respondents by 
calling the respondents' telephone numbers used in round 1. A person who changes his telephone number, perhaps because he moved to another metropolitan area or residence within the same metropolitan area, would not be included in our sample. Of the 34,029 people who were non-elderly and receiving employer-sponsored health insurance in round 1 of the CTSHS, 11,672 were re-surveyed and were still receiving employer-sponsored health insurance in round 2.

In the appendix we report coefficient estimates of a logit regression that equals one if the round-1 respondent who was eligible from our perspective was re-surveyed and still eligible in round 2. Many of the coefficients are statistically significant, which indicates systematic non-response. As expected, people who we are likely to be relatively transient, and therefore to change telephone numbers during a two-year time period, were less likely to be re-surveyed: people between the ages of 18 and 55, non-married individuals, non-whites, and people with relatively low levels of education and income. People who used medical services relatively intensely in the first round were also less likely to be re-surveyed. Our results on risk selection apply, therefore, only to a relatively stable sub-population; we cannot extrapolate our findings to the general employer-sponsored population. However, it is not obvious, to us at least, whether the systematic non-response biases our risk selection estimates and, if so, whether they are biased toward or against finding favorable risk selection for HMOs. That is, non-respondents who expect to use a relatively large amount of medical services may favor either HMOs or non-HMO plans.

The four key variables for this study are the characterization of HMO and non-HMO plans, the definition of a person who has a choice of plan types, the definition of a person who switches plan types, and a person's estimated medical expenditures. CTSHS respondents were asked to define their plan as being an HMO or not. ${ }^{3}$ This question should result in preferred provider organizations (PPOs) being grouped with non-HMO plans, so we are essentially comparing lightly managed non-HMO plans (indemnity and PPO) versus more strictly managed plans (point-of-service and HMO plans). We estimate the risk selection among the sub-sample who was offered a choice of plan types in the second round of 
the survey. We define those with a choice of plan type as all members of a household in which the main health plan policyholder had the opportunity to select either an HMO plan or a non-HMO from his or her employer. We also consider household members to have choice if one member (usually a spouse) was offered an HMO plan only and another member a non-HMO plan only. A "switcher" is defined as an individual who is enrolled in an HMO plan in round 1 and a non-HMO plan in round 2, or in a non-HMO plan in round 1 and an $\mathrm{HMO}$ in round 2.

The CTSHS records medical service use (e.g., number of physician visits, number of hospital days), but not medical expenditures. Since we want an aggregate measure of medical use, we estimate medical expenditure by applying resource weights per unit of medical care (i.e., unit prices) for each of the reported medical services, as was done in Polsky and Nicholson (2002), Manning et al. (1985) and Goldman et al. (1998). The resource use weights are estimated from the 1996 Medical Expenditure Panel Survey (MEPS), and for both survey rounds we use the non-HMO prices. Sample means and standard deviations are presented in Table 1.

\section{Results}

In Table 2 we categorize respondents according to whether they had a choice of health plan types in each round and the plan they actually chose. The rows of Table 2 refer to the first round and the columns to the second. The first entry of each "cell" reports the mean medical expenditures for those respondents in the first round, the second entry in brackets adjusts these first-round medical expenditures for an enrollee's gender and age, and third entry in brackets indicates the number of respondents in the cell. For example, a total of 1,897 people were offered a non-HMO only plan by their employer in both round 1 and round 2 . These people had actual medical expenditures in round 1 of $\$ 1,878$, on average. Their risk-adjusted medical expenditures were $\$ 1,900$, which indicates that their gender and age mix is similar to that of the overall sample. Our regression analysis will be restricted to people who had a choice

\footnotetext{
${ }^{3}$ If necessary, an interviewer elaborated as follows: "With an HMO, you must generally receive care from HMO doctors; otherwise, the expense is not covered unless you were referred by the HMO or there was a medical
} 
of plan types in round 2 - people in the final two columns of Table 2. People who switch plan types among those offered a choice in round 2 are listed in bold, and the percentage switching among those offered a choice of plan types in round 2 is reported in the final column.

Almost half of the sample had no choice of plan type in round 1. That is, they were offered either a non-HMO only (row 1) or an HMO only (row 2) by their employer in round 1. A number of these people were offered a choice plan types in round 2 (column 3 and column 4 of rows 1 and 2), and a fairly large percentage of these people who were able to switch plan types between round 1 and round 2 did so. For example, 183 of the 726 people (25.2 percent) who were offered an HMO only in round 1 and were offered both types in round 2, switched to a non-HMO. These switchers had medical expenditures in round 1 that were $\$ 1,001$ higher than the people who were also in an HMO in round $1(\$ 2,861-\$ 1,860)$, were also offered a choice in round 2, and chose to remain in an HMO. We measure risk selection with round-1 medical expenditures because the plan design and management were presumably the same in round 1 for those who subsequently switched out of and those who remained in an HMO; differences in medical expenditures should be due to differences in the demand for medical services.

Non-HMOs experience adverse selection among the no-choice group enrolled in an HMO in round 1 . However, this risk selection is based on easily observed enrollee characteristics such as gender and age. The difference in risk-adjusted expenditures between the "switchers" and the "stayers" in the second cell of row 2 is only $\$ 84: \$ 1,910$ - $\$ 1,826$. A simple risk adjustment system could equate the expected profit of these enrollees from a health plan's perspective. However, HMOs experienced adverse selection among the non-HMO enrollees who had no choice of plan type in round 1 but did have a choice in round 2 (row 1, column 3 and column 4 of Table 2). The people switching to an HMO in round 2 had medical expenditures in round 1 that were $\$ 392$ higher than the people who remained in a non-HMO $(\$ 2,020$ - \$1,628). These descriptive measures of risk selection do not control for the respondent's market or control for more difficult to observe enrollee characteristics such as health status and income.

emergency." 
People who were offered a choice of plan types in both rounds (column 3 and 4 of row 3 and 4) are less likely to switch plan types than those who were constrained in round 1, as expected, although more than one-sixth of the people did switch. HMOs experienced favorable selection among non-HMO enrollees who had a choice of plan types in round 1 and round 2 (row 3, column 3 and column 4). Round-1 medical expenditures for these enrollees who switched to an HMO in round 2 were $\$ 529$ lower relative to enrollees who remained in a non-HMO plan $(\$ 1,511-\$ 2,040)$.

We present our risk selection estimates in Table 3 and Table 4. The sample for the regressions consists of individuals who were interviewed in both rounds of the survey and were offered both an HMO and a non-HMO in the second round. In the first column of Table 3 we regress an individual's medical expenditures in the first round on four indicator variables that describe a person's plan in round 1 and round 2 and a set of 60 indicator variables for a person's market (usually an MSA). We do not include employee characteristics in the first specification in order to measure risk selection in a situation where firms engage in no formal or informal risk adjustment of premiums. Coefficients on the plan indicator variables that appear in bold are statistically different from one another at the 10-percent level, and provide evidence that the expenditures of people who switched plan types differed from those who remained, on average.

People who switched from a non-HMO to an HMO plan and had a choice of plan types in the first round used $\$ 210$ fewer medical resources in the first round $(\$ 1,320-\$ 1,530)$ than those who remained in a non-HMO plan (column 1 of Table 3). However, this difference is not statistically significant. HMOs, on the other hand, did experience favorable selection because the enrollees who switched to non-HMO plans in the second round used more medical resources than those who remained in an HMO. HMO enrollees who switched to a non-HMO in round 2 used $\$ 370$ more medical services in round $1(\$ 1,957-\$ 1,587)$, or 18 percent of the mean medical expenditures of HMO enrollees in round 1.

In the second column of Table 3 we add indicator variables for gender and age to see if the differences in medical utilization between those who do and do not switch plan types are due to differences in enrollee characteristics that an employer could use to adjust premiums. If those switching 
from a non-HMO to an $\mathrm{HMO}$ have lower expenditures because they are male and relatively young, characteristics associated with lower predicted medical expenditures, then employers could pay plans relatively low premiums when they enroll these types of workers. Although gender and age do affect first round medical expenditures, as can be seen in column 2 of Table 3, HMOs would have still experienced favorable risk selection in a simple risk-adjusted market. Medical expenditures for the HMO enrollees who switched to a non-HMO are still significantly higher than those who remained $(\$ 2,435$ vs. $\$ 2,058)$, even after controlling for gender and age. This implies that selection occurs based on unobserved preferences for medical care and/or characteristics that are more difficult for employers to observe.

In the third column of Table 3 we include an extensive set of individual and firm characteristics, such as self-reported health status, marital status, household structure (e.g., married with children), race, education, household income, type of employer (i.e., private or government), and number of employees at the person's firm. When we include these characteristics that may be difficult for a firm to observe in order to control for enrollee expenditures, neither plan type would experience risk selection.

Does the favorable risk selection for HMOs that we detect have a substantial impact on health insurers' financial performance? In our sample there were 609 people who switched from an HMO to a non-HMO. Based on the second specification from Table 3, these people who switched to a non-HMO used $\$ 377$ more medical expenditures in the initial year than those who remained in an HMO, or 18 percent more medical resources than the average HMO enrollee. These 609 people represent 10 percent of HMO enrollment in round 1 of our sample, so the loss of these relatively expensive HMO enrollees would be predicted to decrease aggregate HMO medical expenditures by 2.0 percent. Although the annual impact appears to be small, if this magnitude of risk selection occurred over an extended time period, it could presumably allow HMOs to charge lower premiums than non-HMO plans and attract employees who would prefer to enroll in an non-HMO plan under a perfect risk adjustment scheme.

In Table 4 we repeat the analysis presented in Table 3 but we now include eight indicator variables to examine risk selection separately for people who did and did not have a choice of plan types in the first round of the survey. We would expect risk selection to be more pronounced for people who 
were initially constrained - their employer offered either an HMO only or a non-HMO only. We find some evidence supporting this hypothesis. According to column 1 of Table 4, people who switched from a non-HMO plan and had a choice of plan types in the first round used $\$ 526$ fewer medical resources in the first round $(\$ 1,121-\$ 1,647)$ than those who remained in the non-HMO plan. This difference, which is 28 percent of the non-HMO average expenditure, is statistically significant at the 5-percent level and economically significant as well.

The favorable selection for HMOs that we report in Table 3 appears to be concentrated among people who were offered an HMO only in the first round of the survey. Among people who had no choice of plan types in round 1 and were in an HMO, those who switched to a non-HMO in round 2 used $\$ 1,000$ more medical services in round $1(\$ 2,475-\$ 1,475)$. In the second column of Table 4 , when we control for an enrollee's gender and age, the adverse selection experienced by non-HMOs and the favorable selection experienced by HMOs would persist. When we include a more extensive set of risk adjusters in column 3 of Table 4, HMOs would still experience favorable selection among the enrollees who did not have a choice of plan types in the first round.

Our analysis indicates that people switching from HMOs to non-HMOs tend to have relatively high medical expenditures. However, if this expenditure difference is not persistent, then risk selection may not affect health plan profits and may not cause distortions in plan design. This would be the case if the switchers' deviations from average expenditures are due to transitory health shocks that do not persist in the following years. In Table 5 we present coefficient estimates of the determinants of medical resource use in the second round of the survey. The structure of the regressions is similar to Table 3 and the sample consists, as before, of people who had a choice of plan types in the second round.

People who switched from an HMO in round 1 to a non-HMO in round 2 used $\$ 119$ more $(\$ 1,421$ - $\$ 1,302)$ medical resources in round 2 than the non-HMO enrollees who remained in a nonHMO. This expenditure difference is not significant in column 1, or in the other columns when we control for enrollees' personal characteristics. Therefore, we cannot reject the hypothesis that the enrollees that non-HMO plans acquire from HMOs have the same expenditures as the experienced non- 
HMO enrollees once they join the non-HMO plan. One explanation for this is a regression to the mean: these people had an acute illness in the initial period, used a relatively large amount of medical resources in the HMO, became disenchanted with the HMO and switched to a non-HMO in the second period, and used an average amount of medical resources in the non-HMO when their health returned to its normal state.

The non-HMO enrollees who switch to an HMO, on the other hand, do use \$322 fewer medical resources $(\$ 936$ - \$1,258) in round 2 than the HMO enrollees who remained in an HMO. This difference is statistically significant and remains significant when we control for age and gender, which indicates that the expenditure difference is due to unobserved enrollee characteristics such as preferences for medical care or health. Even with a simple risk adjustment system, HMOs would appear to be able to benefit from favorable risk selection. This is a somewhat surprising result because overall, across the choice and no-choice groups, the non-HMO enrollees who switched to an HMO did use \$200 fewer medical resources than the enrollees who remained in a non-HMO, but this difference was not statistically significant. Apparently the people who switched to an HMO have persistently lower expenditures, possibly because they have weaker preferences for medical care and/or their health is positively and strongly correlated over time. The expenditure difference is slightly smaller in magnitude and statistically insignificant in the final specification where we include variables that perhaps could not be incorporated into a feasible risk adjustment system.

Our results, from a national data set, are generally consistent with switcher studies that have used a single employer or a coalition of employers in a single market, although the magnitude of our effects are smaller. Altman, Cutler, and Zeckhauser (1998), for example, find that people switching from an indemnity to an HMO plan spent 36 percent less on medical care than those remaining in the indemnity plan, and people switching from an HMO to an indemnity plan spent 47 percent more than those who remained in an HMO. Jackson-Beeck and Kleinman (1983) find that people enrolling in an HMO when it was offered for the first time had an average of 53 percent fewer hospital days in the indemnity plan relative to people who remained in the indemnity plan. 
Our finding of favorable risk selection for HMOs differs from the RAND study and the study by Polsky and Nicholson (2002), studies that estimate average rather than marginal risk selection measures. As part of the RAND Health Insurance Experiment, people were randomized to a Seattle HMO and their medical utilization was compared to people who chose to enroll in the same HMO. Differences in utilization would be due, therefore, to differences in the demand for medical care rather than plan design. Manning et al. (1987) found no difference in the use of medical services between the two groups. Likewise, Polsky and Nicholson (2002) found no evidence of risk selection using the first wave of the CTSHS. They decompose differences in expenditures between HMO and non-HMO enrollees into a utilization, reimbursement, and risk selection effect, where the latter effect is measured as a residual. The switcher methodology generates a marginal risk selection estimate, since we identify the risk selection coefficients by the people who switch plan types. If the people who remain in an HMO, for example, use slightly more medical services than people who remain in a non-HMO, then the average risk selection measure may in fact be zero.

Another way to analyze risk selection is to see whether non-HMO enrollees with relatively high medical expenditures in round 1 are more likely to switch to an HMO in round 2, and if HMO enrollees with relatively low medical expenditures in round 1 are more likely to switch to a non-HMO in round 2 (Call et al., 1999). We examine this issue with descriptive data in Table 6 and with a regression analysis in Table 7. In the first panel of Table 6 we divide the non-HMO enrollees in round 1 that had a choice of plan types in round 2 (and thus could choose their preferred plan easily) into six categories according to their medical expenditures in round 1. Twenty-nine percent of the non-HMO enrollees who did not use any medical resources in round 1 switched to HMO plans in round 2. In fact, the proportion of non-HMO enrollees who switched does not differ much across the first five expenditure categories. However, the non-HMO enrollees in the top expenditure decile, with average medical expenditures in round 1 of $\$ 11,700$, were much less likely to switch to an HMO (0.234 versus 0.286 for non-HMO enrollees as a whole). This is consistent with the anecdotal stories that people with chronic diseases place a great value on remaining in a health plan that allows greater choice of providers and practices less aggressive 
utilization management. The low likelihood of switching among the heaviest users of medical care seems sensible because people who use a great deal of medical services are more likely than other enrollees to learn whether the plan is a good match.

In the second panel of Table 6 we divide the HMO enrollees in round 1 that had a choice of plan types in round 2 into six categories according to their medical expenditures in round 1. Eighteen percent of these HMO enrollees switched to a non-HMO plan in the second round. Interestingly, the HMO enrollees who did not use any medical resources in round 1 were most likely to switch $(0.226$ versus and average of 0.184). These enrollees may have switched in part because they were dissatisfied with the provider network and/or the plan rules. This instance of favorable selection for non-HMOs is counteracted by adverse selection at the other end of the HMO expenditure distribution. HMO enrollees in the top decile, who had mean round-1 medical expenditures of $\$ 12,500$, were more likely to switch to a non-HMO plan than the average HMO enrollee (0.210 versus 0.184$)$.

In Table 7 we present results of probit regressions where the dependent variable is one if a person switches plan types between round 1 and round 2, and is a zero otherwise. In the first two columns we restrict the sample to people enrolled in a non-HMO plan in the first round who also had a choice of plan types in the second round. In the first column we include an indicator variable for individuals who did not use any medical care in the first round, a continuous variable of medical expenditures in the first round, and expenditures squared. None of these coefficients is significant. This is consistent with Table 6, which shows that the proportion of people switching to an HMO was not correlated with use of medical resources except for enrollees who had about $\$ 10,000$ of medical expenditures in the first round, and is also consistent with the results from Table 3, where the non-HMO enrollees switching to HMOs did not have statistically different expenditures than those remaining in a non-HMO.

When we include indicator variables for a person's gender and age in column 2, the coefficients on medical expenditures now indicate whether people who used more medical resources than would be predicted based on these observable characteristics, were more likely to switch to an HMO. Such would be the case, for example, if non-HMO enrollees with strong preferences for medical care and poor health 
used a lot of medical care and switched to HMO plans. The insignificant coefficients on the medical expenditure variables in column 1 and column 2 indicate that medical use is not strongly correlated with the likelihood that non-HMO enrollees will switch to an HMO plan. The positive coefficients on the age variables in column 2 demonstrate that relatively young non-HMO enrollees switched to HMO plans in the second period.

In column 3 and column 4 of Table 7 we present results of probit regressions for the sample of people who were enrolled in an HMO in round 1 and had a choice of plan types in round 2. The positive coefficient on the medical expenditures in column 4 indicates that non-HMOs are experiencing adverse risk selection, and this selection is based on characteristics other than gender and age. That is, HMO enrollees who used more medical resources than predicted based on their gender and age were more likely to switch to a non-HMO plan in the second round. However, medical expenditures in round 1 have a fairly small impact on the likelihood that an HMO enrollee will switch to a non-HMO plan. A onestandard deviation increase in medical expenditures $(\$ 4,657)$ is associated with a 2.5 percentage point increase in the predicted probability that an HMO enrollee will switch to a non-HMO plan (from 0.184 to $0.209)$.

The two methods of examining risk selection appear to be producing consistent results. People who use medical resources relatively intensively are slightly more likely to switch from an HMO to a non-HMO plan. Since the distribution of medical expenditures is skewed to the right, this small difference in the likelihood of switching among the heaviest users of medical services can produce fairly large aggregate differences in medical costs between HMOs and non-HMOs, especially if the risk selection occurs over multiple years.

\section{Conclusions}

Almost all of the existing studies that measure risk selection in the non-elderly, employersponsored health insurance market are case studies of a single employer or employer coalition in a single market, and most use data from the 1980s or early 1990s. Since many employers have mitigated the 
incentive for health plans to target low-risk enrollees by self-insuring and/or by offering a variety of health plans from a single insurance company, the results from these case studies may not be representative of national conditions. In this paper we examine whether HMOs experience favorable risk selection in the employer-sponsored market by applying a "switcher" methodology to a national, panel data set of enrollees in employer-sponsored health plans.

We find evidence that HMOs experience favorable selection that would persist even if employers adjusted health plan premium payments for the enrollees' gender and age. People who switched from a non-HMO to an HMO plan used 11 percent fewer medical services in the period prior to switching than people who remained in the non-HMO plan, and that this low use persists once they enroll in an HMO, relative to those already enrolled in an HMO. Furthermore, we find that people who switched from an HMO to a non-HMO plan used 18 percent more medical services in the period prior to switching than people who remained in the HMO plan. This relatively high use rate does persist once they enroll in the non-HMO, but the differential is not statistically significant. Ten percent of HMO enrollees switched to a non-HMO between the first and second round of the CTSHS survey, and their departure is predicted to reduce an HMO's aggregate medical expenditures by 2.0 percent. Although the annual impact appears to be small, if this magnitude of risk selection occurred over an extended time period, it could presumably allow HMOs to charge lower premiums than non-HMO plans and attract employees who would prefer to enroll in an non-HMO plan under a perfect risk adjustment scheme. 


\section{References}

Altman, D., D. Cutler, and R. Zeckhauser, 2003, "Enrollee Mix, Treatment Intensity, and Cost in Competing Indemnity and HMO Plans," Journal of Health Economics 22(1): 23-45.

Brown, R. S. et al., 1993. The Medicare Risk Program for HMOs - Final Summary Report on Findings From the Evaluation. Final report under HCFA contract No. 500-88-0066. Mathematica Policy Research, Inc., Princeton, NJ.

Call, K. T., B. Dowd, R. Feldman, and M. Maciejewski, 1999, "Selection Experiences in Medicare HMOs: Pre-Enrollment Expenditures," Health Care Financing Review 20(4): 197-209.

Cox, D. F., and C. Hogan, 1997, "Biased Selection and Medicare HMOs: Analysis of the 1989-1994 Experience, Medical Care Research and Review 54: 259-274.

Cutler, D. M., and R. J. Zeckhauser, 1998, “Adverse Selection in Health Insurance,” in A. M. Garber, ed., Frontiers in Health Policy Research, Vol. 1. Cambridge, MA: MIT Press.

Eggers, P., 1980, "Risk Differential Between Medicare Beneficiaries Enrolled and Not Enrolled in an HMO," Health Care Financing Review 1(3): 91-99.

Eggers, P., and R. Prihoda, 1982, "Pre-Enrollment Reimbursement Patterns of Medicare Beneficiaries Enrolled in 'At Risk' HMOs," Health Care Financing Review 4(1): 5-73.

Goldman, D. P., A. Leibowitz, and J. L. Buchanan, 1998, "Cost-containment and Adverse Selection in Medicaid HMOs," Journal of the American Statistical Association 93: 54-62.

Hill, J. W., and R. S. Brown, 1990. Biased Selection in the TEFRA HMO/CMP Program. Princeton, NJ: Mathematica Policy Research, Inc.

InterStudy, 2000. National Policy and Employer-Based Health Plans: An Analysis and Response From InterStudy Publications. Available at: http://www.hmodata.com/pdf/natpol emp hlthpln.pdf.

Jackson-Beeck, M., and J. H. Kleinman, 1983, "Evidence For Self-Selection Among Health Maintenance Organization Enrollees," Journal of the American Medical Association 250(20): 2826-2829.

Keenan, P. S., M. J. B. Buntin, T. G. McGuire, and J. P. Newhouse, 2001, "The Prevalence of Formal Risk Adjustment in Health Plan Purchasing," Inquiry 38: 245-259.

Kemper, P. S., M.J.B. Buntin, J. M. Corrigan, P. J. Cunningham, S.M. Felt, J. M. Grossman, K. T. Kohn, C. E. Metcalf, R. F. St. Peter, R. C. Strouse, and P. B. Ginsburg, 1996, "The Design of the Community Tracking Study: A Longitudinal Study of Health System Change and Its Effect on People," Inquiry 33: 195-206.

Manning, W. G., J. P. Newhouse, and J. P. Duan, 1987, "Health Insurance and the Demand for Medical Care: Evidence From a Randomized Experiment," American Economic Review 77: 251-277.

Manning, W. G., A. Leibowitz, G. A. Rogers, and J. P. Newhouse, 1985, "A Controlled Trial of the Effect of a Prepaid Group Practice on the Utilization of Medical Services," New England Journal of Medicine 310: 1505-1510. 
McDonnell, K., and P. Fronstin, 1999. EBRI Health Benefits Databook, First Edition. Washington, D.C.: Employee Benefit Research Institute.

Polsky, D., and S. Nicholson, 2002, "Why Are Managed Care Plans Less Inexpensive: Risk Selection, Utilization, or Reimbursement?" Journal of Risk and Insurance, forthcoming. 
Table 1

Sample Means and Standard Deviations $(n=11,672)$

$\underline{\text { Round } 1 \text { Variables }}$

Medical resource use (\$000):

- $1^{\text {st }}$ round (1996-1997)

$-2^{\text {nd }}$ round (1998-1999)
Mean

$\$ 1,859$

$\$ 1,644$

$\underline{\text { Standard Deviation }}$

Individual and firm characteristics from round 1:

Age

Under 2 years

2-17

18-39

$40-55$

56-64

Female

Marital status:

- single, with children

- single, no children

- married, no children

- married, with children

Race:

White

African American

Asian

Hispanic

Education:

Less than high school

High school graduate

College graduate

Masters degree

Self-reported health:

- excellent

- very good

- good

- fair

- poor

Household income:

- below $\$ 10,000$

- \$10,000 - \$49,999

- $\$ 50,000-\$ 99,999$

- \$100,000+

Employer:

- government

- fewer than 10 employees

- 10-1000 employees

- 1000+ employees
0.017

0.188

0.340

0.367

0.087

0.526

0.071

0.130

0.210

0.590

0.843

0.071

0.031

0.055

0.242

0.264

0.383

0.111

0.347

0.398

0.197

0.049

0.009

0.051

0.431

0.430

0.088

0.219

0.102

0.325

0.355
$\$ 4,317$

$\$ 3,883$
0.129

0.391

0.474

0.482

0.283

0.499

0.256

0.336

0.407

0.492

0.364

0.257

0.174

0.228

0.428

0.441

0.486

0.315

0.476

0.490

0.398

0.215

0.094

0.220

0.495

0.495

0.283

0.413

0.302

0.468

0.479 
Table 2

Health Plan Offerings, Choices, and Medical Use in Round 1 and Round 2 of the CTSHS Survey

Top entry:

mean medical use in Round 1

Second entry: risk-adjusted (for age and gender) medical use in Round 1

Third entry: [number of enrollees]

People who switched plan types and had a choice of plans in Round 2 are in bold.

\section{Health Plan in Round 2}

\section{Health Plan in Round 1}

Non-HMO, not offered choice

$\$ 1,878$
$\$ 1,900$
$[n=1,897]$

$\$ 1,349$
$\$ 1,788$
$[n=493]$

$\$ 1,715$
$\$ 1,829$
$[n=293]$

Non-HMO,
No Choice

HMO,

Non-HMO,

HMO,

No Choice

Choice

Choice

$\underline{\text { Total }}$

Percentage Switching Plan Types if Offered a Choice in Round 2

\section{(a)}

HMO, not offered choice

Non-HMO, offered choice

HMO, offered choice

$$
\begin{aligned}
& \$ 1,677 \\
& \$ 1,879 \\
& {[n=178]}
\end{aligned}
$$

$\$ 1,525$
$\$ 1,804$
$[n=684]$

$\$ 1,628$

$[\mathrm{n}=550]$

$\$ 2,020$
$\$ 1,870$
$[n=325]$

$\$ 1,782$

$[\mathrm{n}=684]$

$\$ 2,861$

$$
\begin{array}{r}
\$ 1,820 \\
\$ 1,839 \\
{[n=1,223]}
\end{array}
$$

$\$ 1,910$

$[\mathrm{n}=183$ ]

$\$ 1,860$

$\$ 1,826$

$[\mathrm{n}=543]$

$\$ 1,860$

$$
[\mathrm{n}=3,456]
$$

$37.1 \%$

$$
\begin{aligned}
& \$ 2,057 \\
& \$ 1,830 \\
& {[n=188]}
\end{aligned}
$$

\section{$\$ 2,040$}

$\$ 1,888$

$[\mathrm{n}=1,546]$

$\$ 1,511$

$\$ 1,811$

$[\mathrm{n}=513]$

$\$ 1,812$

$\$ 1,831$

$\$ 1,619$

$\$ 1,894$

$\$ 2,121$

$\$ 1,848$

$[\mathrm{n}=481]$

$[\mathrm{n}=426]$

$\$ 2,009$
$\$ 1,880$
$[n=2,149]$

$\$ 2,024$

$\$ 1,862$

$\$ 1,873$

$[\mathrm{n}=2,861]$

\section{$\$ 1,722$}

$\$ 1,839$

$[\mathrm{n}=2,576]$

$[\mathrm{n}=2,705]$

$[\mathrm{n}=2,442]$

$25.2 \%$

$24.9 \%$

$\$ 1,861$

$[\mathrm{n}=2,540]$

$16.5 \%$ 
Table 3: Risk Selection Based on Medical Care Use in Round 1

\begin{tabular}{|c|c|c|c|c|c|c|}
\hline Variable & Coefficient & $\begin{array}{l}\text { Std. } \\
\text { Error }\end{array}$ & Coefficient & $\begin{array}{l}\text { Std. } \\
\text { Error }\end{array}$ & Coefficient & $\begin{array}{l}\text { Std. } \\
\text { Error }\end{array}$ \\
\hline
\end{tabular}

Non-HMO in round 1

- non-HMO in round 2

- HMO in round 2

$1530^{* *}$

$1320 * *$

466

482

$1992 * *$

$1803^{* *}$

495

503

943

860

695

HMO in round 1

- HMO in round 2

- non-HMO in round 2

$1587^{* *}$
$1957^{* *}$

472

489

$\begin{array}{rr}\mathbf{2 0 5 8}^{* *} & 508 \\ \mathbf{2 4 3 5}^{* *} & 525 \\ & \\ 632^{* *} & 110 \\ & \\ 3934^{* *} & 700 \\ -1445^{* *} & 264 \\ -766^{* *} & 274 \\ -865^{* *} & 270\end{array}$

961

1249

702

Female

Age: (56-65 is omitted group)

- under 2 years of age

- between 2 and 17

- between 18 and 40

- between 40 and 55

Other potentially observable

characteristics included?

Number of observations

Notes: dependent variable is medical care use in round 1 , measured in dollars. $* *=$ significantly different from zero at the five-percent level; $*=$ significantly different from zero at the 10-percent level. Bold coefficients indicate that the expenditure difference in round 1 between the people switching plan types and those remaining in a particular plan type is significantly different from zero at the 10-percent level. All specifications include indicator variables for the site of the survey (usually a metropolitan statistical area). Additional potentially observable enrollee and employer characteristics include: self-reported health, marital status, children, race, education, household income, type of employer (i.e., private or government), and number of employees at the person's firm. The constant is omitted. Sample includes people who had a choice between an $\mathrm{HMO}$ and a non-HMO plan in round 2. 
Table 4: Risk Selection Based on Medical Care Use in Round 1 for Enrollees With and Without a Choice of Plan Types in Round 1

\section{$\underline{\text { Variable }}$ \\ $\begin{array}{cc}\text { Std. } \\ \text { Coefficient } & \underline{\text { Error }}\end{array}$ \\ $\underline{\text { Coefficient }}$ \\ Std. \\ Error \\ $\underline{\text { Coefficient }}$ \\ Std.}

Non-HMO, no choice in round 1

- non-HMO in round 2

- HMO in round 2

Non-HMO, with choice in round 1

- non-HMO in round 2

- HMO in round 2

HMO, no choice in round 1

- HMO in round 2

- non-HMO in round 2

HMO, choice in round 1

- HMO in round 2

- non-HMO in round 2

Female

Age: (56-65 is omitted group)

- under 2 years of age

- between 2 and 17

- between 18 and 40

- between 40 and 55

Other potentially observable characteristics included?

Number of observations

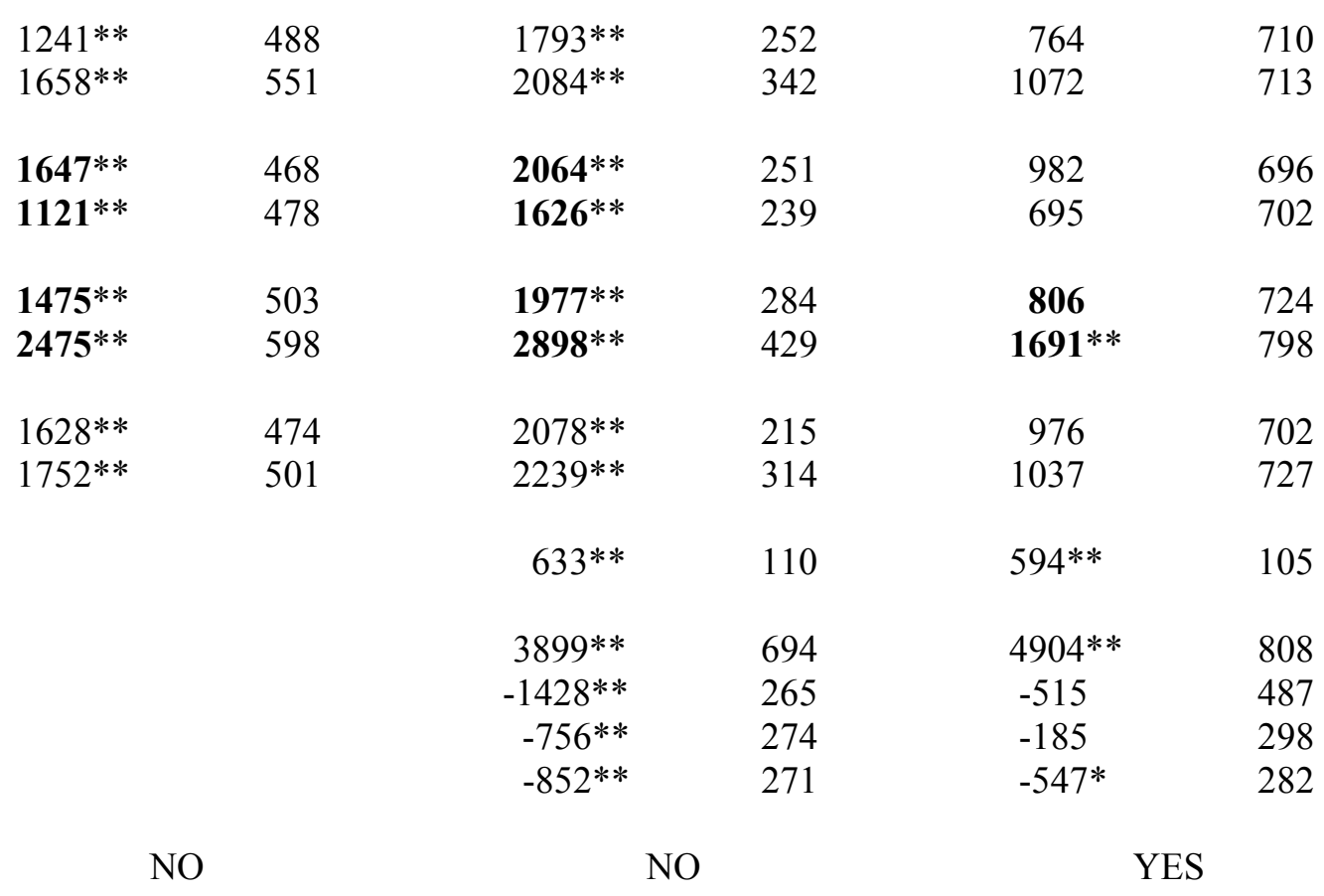

6,235

Notes: dependent variable is medical care use in round 1 , measured in dollars. $* *=$ significantly different from zero at the five-percent level; * $=$ significantly different from zero at the 10-percent level. Bold coefficients indicate that the expenditure difference in round 1 between the people switching plan types and those remaining in a particular plan type is significantly different from zero at the 5-percent level. All specifications include indicator variables for the site of the survey (usually a metropolitan statistical area). Additional potentially observable enrollee and employer characteristics include: self-reported health, marital status, children, race, education, household income, type of employer (i.e., private or government), and number of employees at the person's firm. The constant is omitted. Sample includes people who had a choice between an HMO and a non-HMO plan in round 2. 
Table 5: Coefficient Estimates on the Determinants of Medical Care Use in Round 2 of CTSHS Survey

\begin{tabular}{|c|c|c|c|c|c|}
\hline Variable & Coefficient & $\begin{array}{l}\text { Std. } \\
\text { Error }\end{array}$ & Coefficient & $\begin{array}{l}\text { Std. } \\
\text { Error }\end{array}$ & Coefficient \\
\hline
\end{tabular}

Enrolled in non-HMO in round 2

- non-HMO in round 1

- HMO in round 1 (switcher)

$1302 * *$

$1421 * *$

240

281

$1749^{* *}$

$1917 * *$

323

405

795

851

549

Enrolled in $\mathbf{H M O}$ in round 2

- HMO in round 1

$\begin{array}{rr}\mathbf{1 2 5 8}^{* *} & 232 \\ \mathbf{9 3 6}^{* *} & 252\end{array}$

$1751 * *$

318

1431** 332

722

515

577

- non-HMO in round 1 (switcher)

$552^{* *}$
-228
$-1201^{* *}$
$-832^{* *}$
$-715^{* *}$

$532 * *$

572

Female

Age: (56-65 is omitted group)

- under 2 years of age

- between 2 and 17

- between 18 and 40

- between 40 and 55

Other potentially observable

characteristics included?

Notes: dependent variable is medical care use in round 2, measured in dollars. $* *=$ significantly different from zero at the five-percent level; $*=$ significantly different from zero at the 10-percent level. Bold coefficients indicate that the expenditure difference in round 2 between the people switching plan types and those remaining in a particular plan type is significantly different from zero at the 5-percent level. All specifications include indicator variables for the site of the survey (usually a metropolitan statistical area). Additional potentially observable enrollee characteristics include: self-reported health, marital status, children, race, education, and household income. The constant is omitted. Sample includes people who had a choice between an HMO and a non-HMO plan in round 2. 
Table 6

Probability of Switching Plan Types By Type of Plan and Medical Expenditures in Round 1

Enrolled in a non-HMO plan in Round $1(n=2,934)$

Medical Expenditures in Round 1

$\begin{array}{lccccccc} & \text { Zero } & \begin{array}{l}\text { Zero to } 25^{\text {th }} \\ \text { percentile }\end{array} & \begin{array}{c}25^{\text {th }} \text { to } 50^{\text {th }} \\ \text { percentile }\end{array} & \begin{array}{c}50^{\text {th }} \text { to } 75^{\text {th }} \\ \text { percentile }\end{array} & \begin{array}{c}75^{\text {th }} \text { to } 90^{\text {th }} \\ \text { percentile }\end{array} & \begin{array}{c}\text { Above } 90^{\text {th }} \\ \text { percentile }\end{array} & \begin{array}{c}\text { Total } \\ \text { Mean round 1 expenditures }\end{array} \\ \begin{array}{l}\text { percen } \\ \text { Proportion enrolled in an } \\ \text { HMO plan in round 2 }\end{array} & 0.290 & \$ 203 & \$ 486 & \$ 1,029 & \$ 2,327 & \$ 11,679 & \$ 1,868 \\ & & 0.289 & 0.299 & 0.282 & 0.295 & 0.234 & 0.286 \\ \end{array}$

Enrolled in an HMO plan in Round $1(n=3,301)$

Medical Expenditures in Round 1

\begin{tabular}{|c|c|c|c|c|c|c|c|}
\hline & Zero & $\begin{array}{l}\text { Zero to } 25^{\text {th }} \\
\text { percentile }\end{array}$ & $\begin{array}{l}25^{\text {th }} \text { to } 50^{\text {th }} \\
\text { percentile }\end{array}$ & $\begin{array}{l}50^{\text {th }} \text { to } 75^{\text {th }} \\
\text { percentile }\end{array}$ & $\begin{array}{l}75^{\text {th }} \text { to } 90^{\text {th }} \\
\text { percentile }\end{array}$ & $\begin{array}{l}\text { Above } 90^{\text {th }} \\
\text { percentile }\end{array}$ & Total \\
\hline Mean round 1 expenditures & $\$ 0$ & $\$ 201$ & $\$ 488$ & $\$ 1,070$ & $\$ 2,579$ & $\$ 12,548$ & $\$ 2,046$ \\
\hline $\begin{array}{l}\text { Proportion enrolled in a } \\
\text { non-HMO plan in round } 2\end{array}$ & 0.226 & 0.180 & 0.175 & 0.163 & 0.190 & 0.210 & 0.184 \\
\hline
\end{tabular}

Notes: The sample is restricted to people with a choice of both an HMO and a non-HMO plan in round 2. 
Table 7: Probit Coefficient Estimates: Likelihood of Switching Plan Types

\section{Variable}

No medical use in Round 1

Round 1 medical expenditures $(\$ 000)$

Round 1 expenditures squared

Female

Age: (56-65 is omitted group)

- under 2 years of age

- between 2 and 17

- between 18 and 40

- between 40 and 55

\section{Constant}

Pseudo $\mathrm{R}^{2}$

Number of observations

\section{Enrolled in a Non-HMO in Round 1}

$\begin{array}{ll}-0.169 & -0.0066 \\ (0.0732) & (0.0745) \\ & \\ -0.0191 & -0.0196 \\ (0.0120) & (0.0123) \\ & \\ 3.7 \times 10^{-7} & 4.0 \times 10^{-7} \\ \left(3.4 \times 10^{-7}\right) & \left(3.5 \times 10^{-7}\right)\end{array}$

0.0333

(0.0502)

$0.463^{* *}$

$(0.231)$

$0.375^{* *}$

(0.096)

$0.469 * *$

(0.089)

$0.152 *$

(0.087)

$\begin{array}{ll}-0.537 * * & -0.834 * * \\ (0.0324) & (0.085)\end{array}$

0.001

2,934
0.01

2,934
Enrolled in an HMO in Round 1

$\begin{array}{lc}0.217^{* *} & 0.205^{* *} \\ (0.0772) & (0.0784) \\ & \\ 0.025^{* *} & 0.026^{* *} \\ (0.010) & (0.011) \\ & \\ -4.0 \times 10^{-7} & -4.3 \times 10^{-7} \\ \left(2.6 \times 10^{-7}\right) & \left(2.7 \times 10^{-7}\right) \\ & -0.0252 \\ & (0.0519) \\ & -0.136 \\ & (0.222) \\ & -0.0615 \\ & (0.112) \\ & 0.0190 \\ & (0.104) \\ & 0.0100 \\ & (0.104) \\ & -0.952^{* *} \\ 0.968^{* *} & (0.101) \\ (0.0332) & 0.004 \\ 0.004 & 3,301 \\ 3,301 & \end{array}$

Notes: dependent variable is one if respondent was enrolled in a non-HMO plan in round 1 and an HMO in round 2, or was enrolled in an HMO plan in round 1 and a non-HMO plan in round 2 of the survey. Sample is restricted to people with a choice of both an HMO and a non-HMO plan in round $2 . * *=$ significantly different from zero at the five-percent level; $*=$ significantly different from zero at the 10 -percent level. 
Appendix

Analysis of Response Bias for Round 2 of the Community Tracking Study Household Survey

Dependent variable is one if the person was re-interviewed in round 2, and zero otherwise

\begin{tabular}{|c|c|c|}
\hline & $\underline{\text { Coefficient }}$ & Standard Error \\
\hline Enrolled in an HMO & $-0.153 * *$ & 0.034 \\
\hline Offered HMO and non-HMO & -0.027 & 0.035 \\
\hline HMO * offered both plan types & $0.079^{*}$ & 0.047 \\
\hline Age indicator: $18-39$ & $-0.691 * *$ & 0.040 \\
\hline Age indicator: $40-55$ & $-0.159 * *$ & 0.039 \\
\hline Female & $0.062 * *$ & 0.024 \\
\hline \multicolumn{3}{|l|}{ Marital status: } \\
\hline - single, with children & $-0.405^{* *}$ & 0.046 \\
\hline - single, no children & $-0.446 * *$ & 0.036 \\
\hline - married, no children & $-0.193 * *$ & 0.033 \\
\hline African American & $-0.369 * *$ & 0.044 \\
\hline Asian & $-0.416^{* *}$ & 0.066 \\
\hline Hispanic & $-0.408 * *$ & 0.049 \\
\hline High school graduate & $0.530 * *$ & 0.046 \\
\hline College graduate & $0.476^{* *}$ & 0.045 \\
\hline Masters degree & $0.403 * *$ & 0.054 \\
\hline \multicolumn{3}{|l|}{ Self-reported health: } \\
\hline - very good & $0.133^{* *}$ & 0.028 \\
\hline - good & $0.096 * *$ & 0.034 \\
\hline - fair & 0.028 & 0.055 \\
\hline - poor & 0.096 & 0.112 \\
\hline \multicolumn{3}{|l|}{ Household income: } \\
\hline - below $\$ 10,000$ & $-0.248 * *$ & 0.056 \\
\hline - $\$ 10,000$ - $\$ 49,999$ & $-0.252 * *$ & 0.043 \\
\hline - $\$ 50,000$ - $\$ 99,999$ & 0.006 & 0.040 \\
\hline \multicolumn{3}{|l|}{ Employer: } \\
\hline - government & 0.051 & 0.033 \\
\hline - fewer than 10 employees & $-0.088^{* *}$ & 0.041 \\
\hline - 10-1000 employees & -0.036 & 0.029 \\
\hline $\begin{array}{l}\text { Medical resource use in } \\
\text { round } 1\end{array}$ & $-1.6 \times 10^{-5 * *}$ & $3.22 \times 10^{-0}$ \\
\hline Constant & $-0.362 * *$ & 0.053 \\
\hline $\mathrm{n}$ & \multicolumn{2}{|c|}{34,029} \\
\hline Pseudo $\mathrm{R}^{2}$ & \multicolumn{2}{|c|}{0.03} \\
\hline
\end{tabular}

Notes: $* *=$ significantly different from zero at the five-percent level; $*=$ significantly different from zero at the 10-percent level. 